\title{
BMJ Open Association of brain amyloidosis with the incidence and frequency of neuropsychiatric symptoms in ADNI: a multisite observational cohort study
}

\author{
Naira Goukasian (D , ${ }^{1}$ Kristy S. Hwang, ${ }^{2}$ Tamineh Romero, ${ }^{3}$ Jonathan Grotts, ${ }^{3}$ \\ Triet M. Do, ${ }^{4}$ Jenna R. Groh, ${ }^{5}$ Daniel R. Bateman, ${ }^{6}$ Liana G. Apostolova ${ }^{5}$
}

To cite: Goukasian N, Hwang KS, Romero T, et al. Association of brain amyloidosis with the incidence and frequency of neuropsychiatric symptoms in ADNI: a multisite observational cohort study. BMJ Open 2019;9:e031947. doi:10.1136/ bmjopen-2019-031947

- Prepublication history and additional material for this paper are available online. To view these files, please visit the journal online (http://dx.doi. org/10.1136/bmjopen-2019031947).

Received 27 May 2019 Revised 08 0ctober 2019 Accepted 28 October 2019

Check for updates

(C) Author(s) (or their employer(s)) 2019. Re-use permitted under CC BY-NC. No commercial re-use. See rights and permissions. Published by BMJ.

For numbered affiliations see end of article.

Correspondence to Dr Liana G. Apostolova; lapostol@iu.edu

\section{ABSTRACT}

Objective To investigate the relationship between amyloid burden and frequency of existing and incidence of new neuropsychiatric symptoms (NPS) in elderly with and without cognitive decline.

Methods 275 cognitively normal controls (NC), 100 subjective memory complaint (SMC), 559 mild cognitive impairment (MCl) and 143 Alzheimer's disease dementia subjects from the Alzheimer's Disease Neuroimaging Initiative received $\left({ }^{18} \mathrm{~F}\right)$-florbetapir positron emission tomography (PET) scans. Yearly neuropsychiatric inventory (Neuropsychiatric Inventory (NPI)/NPI-Questionnaire) data were collected from the study partners at each visit. Mean standard uptake volume ratios (SUVR) normalised to whole cerebellum were obtained. Positive amyloid PET scan was defined as mean SUVR $\geq 1.17$. Fisher's exact test was used to compare frequency and incidence between amyloid positive and amyloid negative subjects. Survival analyses were used to estimate of neuropsychiatric symptoms (NPS) between amyloid positive and amyloid negative subjects. Survival analyses were used to estimate hazard ratios for developing the most common NPS by amyloid status. Results No differences in NPS frequency were seen between amyloid positive and amyloid negative NC, $\mathrm{SMC}, \mathrm{MCl}$ or dementia groups. $\mathrm{MCl}$ subjects with amyloid pathology however tended to have greater frequency $x$ severity ( $F x S$ ) of anxiety, hallucinations, delusions, apathy, disinhibition, irritability, aberrant motor behavior, and appetite, but not agitation, depression, night-time disturbances, or elation. $\mathrm{MCl}$ subjects with amyloid pathology were at greater risk for developing apathy, anxiety and agitation over time. Baseline presence of agitation and apathy and new onset agitation, irritability and apathy predicted faster conversion to dementia among $\mathrm{MCl}$ subjects.

Conclusions Amyloid pathology is associated with greater rate of development of new NPS in MCl. Anxiety and delusions are significant predictors of amyloid pathology. Agitation, irritability and apathy are significant predictors for conversion from $\mathrm{MCl}$ to dementia.

\section{BACKGROUND}

Alzheimer's disease (AD) is the most common form of dementia, accounting for $60 \%$ to $80 \%$ of all dementia cases. $\mathrm{AD}$ is characterised
Strength and limitations of this study

- Our analyses used Alzheimer's Disease Neuroimaging Initiative (ADNI) data; ADNI - the premier longitudinal biomarker study in Alzheimer's disease (AD), employs standardised subject assessment and MRI and positron emission tomography scan collection protocols and meticulous data quality control across all study sites.

- A strength of our study is the relatively large sample size and the inclusion of all diagnostic groups across the AD spectrum including cognitively normal participants many of which are in the presymptomatic $A D$ stages, as well as the use of neuropsychiatric data collection tools that are administered to the caregivers and not the subject themselves.

- One of the limitations of this study is that the NeuropsychiatricInventory (NPI) and NPIQuestionnaire use structured questions focused on the frequency and severity of symptoms from the preceding month only. Therefore, intermittent neuropsychiatric symptoms that were not manifested by the subjects in the prespecified timeframe are not adequately captured.

- Another limitation to our study is that ADNI employs rigorous exclusion criteria typical of clinical trials and hence our study cohort might not be representative of the general population.

- ADNI excludes subjects with preexisting depression (Geriatric Depression Scale score $>5$ ) which likewise could affect the generalisation of the results.

by cortical amyloid plaque and neurofibrillary tangle deposition, as well as progressive synaptic and neuronal loss. Recently developed amyloid positron emission tomography (PET) radioligands with high affinity for amyloid plaques, such as $\left({ }^{18} \mathrm{~F}\right)$-florbetaben, $\left({ }^{18} \mathrm{~F}\right)$-flutemetamol and $\left({ }^{18} \mathrm{~F}\right)$-florbetapir, can provide reliable in vivo visualisation of cortical fibrillar $\beta$-amyloid plaque deposition. ${ }^{1-3}$ With amyloid accumulation beginning up to two decades prior to symptoms onset, these amyloid PET radiotracers can 


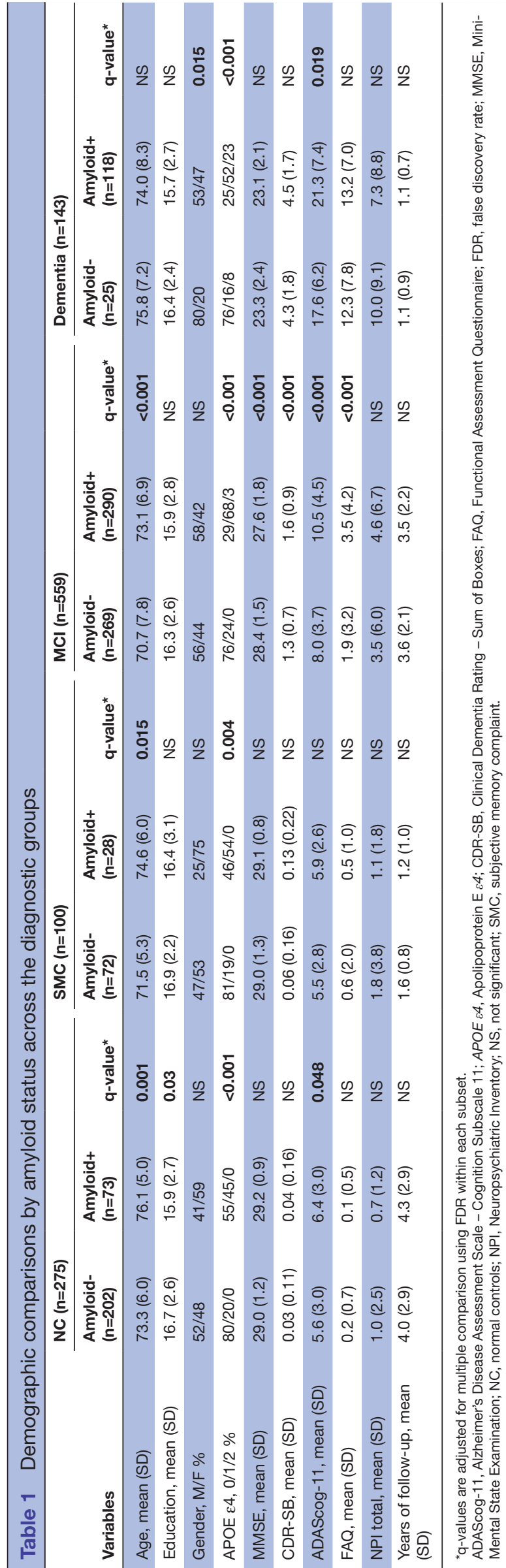

measure amyloid burden in the symptomatic, as well as the asymptomatic stages. ${ }^{45}$

Neuropsychiatric symptoms (NPS) are prominent features of $\mathrm{AD}$ and mild cognitive impairment (MCI) and $35 \%$ to $75 \%$ of MCI subjects experience at least one neuropsychiatric symptom with depression, apathy and anxiety being the most prevalent. ${ }^{6-8}$ In the dementia stage, apathy, agitation and anxiety are most prevalent, followed by aberrant motor behaviour, dysphoria and disinhibition. $^{79}$

NPS have been associated with higher likelihood for cognitive decline. Symptoms of depression, irritability and agitation were found to predict cognitive decline among cognitively normal, MCI, as well as individuals with subjective cognitive concerns pooled together. ${ }^{10}$ One population-based study found that the presence of symptoms such as agitation, apathy, anxiety, irritability and depression in normal controls (NC) at baseline significantly predicted incident MCI. ${ }^{11}$ Neuroticism and its underlying facets of anxiety, depression and stress were associated with diagnosis of MCI and neurodegeneration. ${ }^{12}$ Other studies have shown that the presence of one or more NPS in MCI, specifically symptoms of agitation, depression and apathy, increase the risk of progression from MCI to AD dementia. ${ }^{10-19}$ Most patients with AD experience at least one NPS during the course of disease progression. ${ }^{6}$ NPS in dementia are associated with faster disease progression, ${ }^{20} 21$ increased mortality ${ }^{15}$ and caregiver burden ${ }^{22}$ as well as shorter time until nursing home admission. ${ }^{23} 24$

To date a few groups have studied the associations between NPS and amyloid burden and some have suggested that NPS reflect underlying amyloid pathology. ${ }^{25}{ }^{26}$ Studies have gone on to show that increased apathy was significantly associated with greater cortical amyloid burden in MCI. ${ }^{27}$ Anxious MCI subjects were 3.1 times more likely to have abnormal cerebrospinal fluid amyloid $\beta$ levels - a proxy marker for brain amyloidosis. ${ }^{28}$ Another group found both anxiety and irritability to associate with greater amyloid burden across the $\mathrm{AD}$ spectrum. ${ }^{29}$ Amyloid positive NC who reported anxiety experienced faster cognitive decline ${ }^{30}$ and greater frequency of mood disturbances over the following year. ${ }^{31}$ Taken together these data seem to suggest that at least some NPS are associated with amyloid pathology and cognitive decline.

Here we investigate the relationship between amyloid burden and incidence and frequency of NPS across the spectrum from normal cognition to dementia. We hypothesised that amyloid pathology will associate with higher incidence of NPS across all disease stages. We postulated that amyloid deposition will associate with apathy, anxiety, irritability and depression in the asymptomatic and early symptomatic stages, and with psychosis, aberrant motor behaviours, disinhibition and agitation in the late symptomatic stages. 

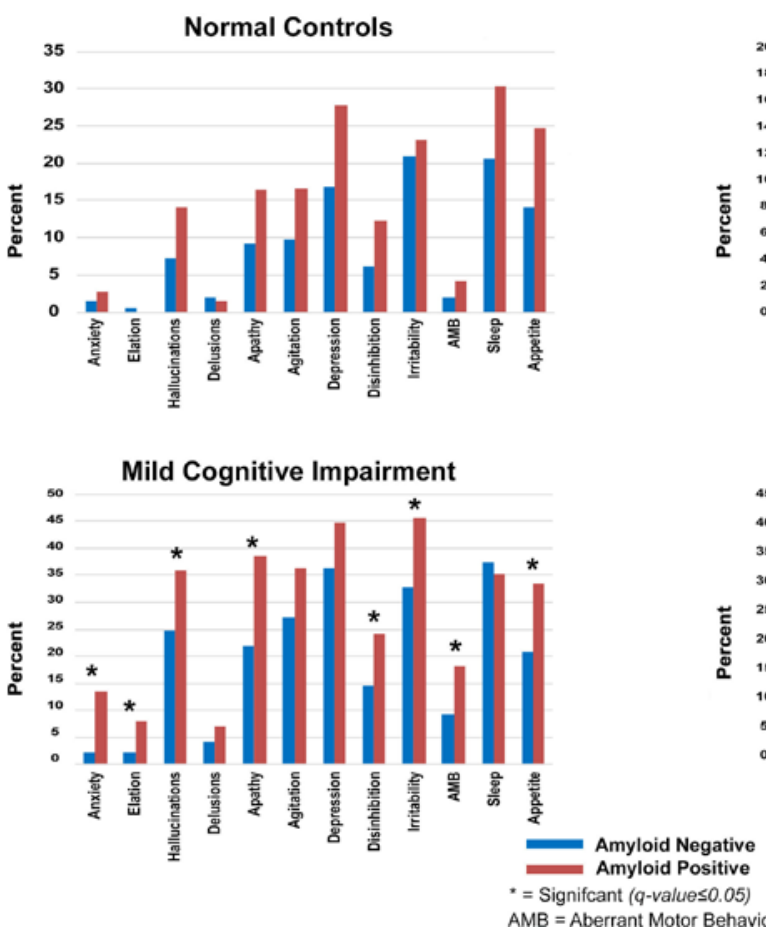

Subjective Memory Complaints
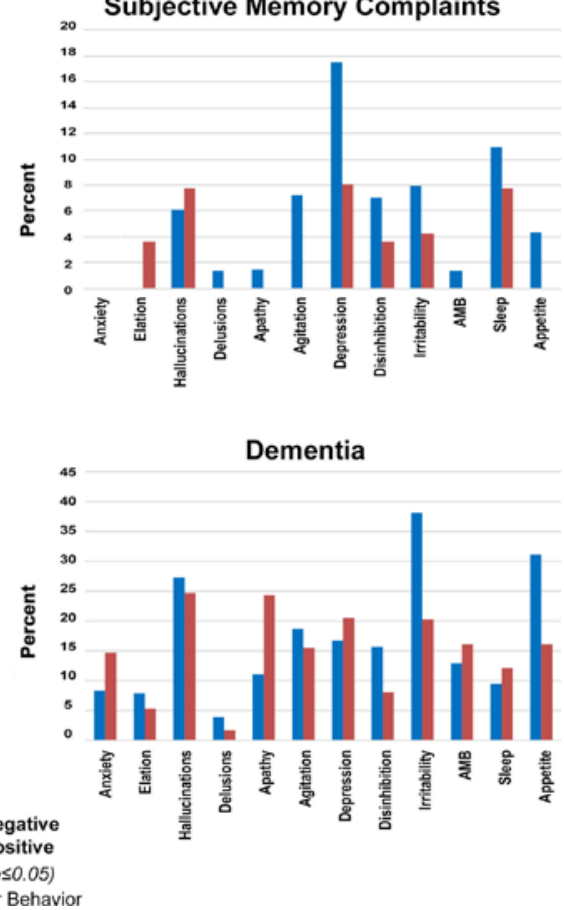

Figure 1 De novo emergence of neuropsychiatricsymptoms by diagnosis and amyloid status. Note: y-axis vary by diagnosis.

\section{METHODS}

\section{Subjects}

Data used in these analyses were obtained from the Alzheimer's Disease Neuroimaging Initiative (ADNI) database (adni.loni.usc.edu). The ADNI was launched in 2003 as a public-private partnership. The primary goal of ADNI has been to test whether serial MRI, PET, other biological markers and clinical and neuropsychological assessment can be combined to measure the progression of MCI and early AD.

The first phase of ADNI, ADNI-1, recruited approximately 800 adults, ages 55 to 90 across 50 sites in the USA and Canada. The study sample consisted of approximately 200 older NC individuals, 400 people with late amnestic MCI (LMCI) and 200 people with mild AD. ADNI expanded enrolment criteria with the launch of ADNI-GO in 2009 and enrolled 200 additional subjects with early amnestic MCI (EMCI). ADNI-2 added approximately 650 newly enrolled subjects ( $150 \mathrm{NC}, 100$ subjects with subjective memory complaints (SMC), 100 EMCI, 150 LMCI and 150 mild AD). All procedures were approved by the Institutional Review Boards of all participating institutions. Written informed consent was obtained from every research participant according to the Declaration of Helsinki and the Belmont Report.

The clinical description of the ADNI cohort has been previously published. ${ }^{32}$ Diagnosis of AD was based on the National Institute of Neurological and Communicative Disorders and Stroke and the AD and Related Disorders Association criteria. ${ }^{33} \mathrm{AD}$ subjects were required to have Mini-Mental State Examination (MMSE) ${ }^{34}$ scores between 20 and 26 and a Clinical Dementia Rating scale (CDR) ${ }^{35}$ score of 0.5 to 1 at baseline. Qualifying MCI subjects had memory complaints, but no significant functional impairment, scored between 24 and 30 on the MMSE, had a global CDR score of 0.5 , a CDR memory score of 0.5 or greater and objective memory impairment on Wechsler Memory Scale - Logical Memory II test. ${ }^{36} \mathrm{NC}$ and SMC subjects had MMSE scores between 24 and 30, a global CDR of 0 and did not meet criteria for MCI and AD. NC were devoid of cognitive concerns, while SMC subjects had significant memory concerns manifested in a score of $\geq 16$ on the first 12 items of the Cognitive Change Index. ${ }^{37}$ Subjects were excluded if they refused or were unable to undergo MRI, had other neurological disorders, active depression or history of psychiatric diagnosis, alcohol or substance dependence within the past 2 years, less than 6 years of education or were not fluent in English or Spanish. Inclusion and diagnostic criteria, as well as procedures and protocols, for the ADNI studies can be found on http://www.adni-info.org/Scientists/ADNIStudyProcedures.html. Written informed consent was obtained from all participants. For more up-to-date information, see www.adni-info.org.

$\left({ }^{18} \mathrm{~F}\right)$-florbetapir PET amyloid imaging was added in the ADNI-GO/2 stages of the study. We downloaded the clinical, behavioural and amyloid PET data of $275 \mathrm{NC}$, $100 \mathrm{SMC}, 559 \mathrm{MCI}$ and $143 \mathrm{AD}$ subjects from the ADNI-1, ADNI-GO and ADNI-2 databases on 3 November, 2015. EMCI and LMCI were grouped in our analyses.

\section{Neuropsychiatric data}

Neuropsychiatric data were captured with the Neuropsychiatric Inventory (NPI) ${ }^{38}$ and the NPI Questionnaire (NPI-Q). ${ }^{39}$ ADNI-1 used the NPI-Q while ADNI-GO/2 used the full version. Both versions assess 12 symptoms 

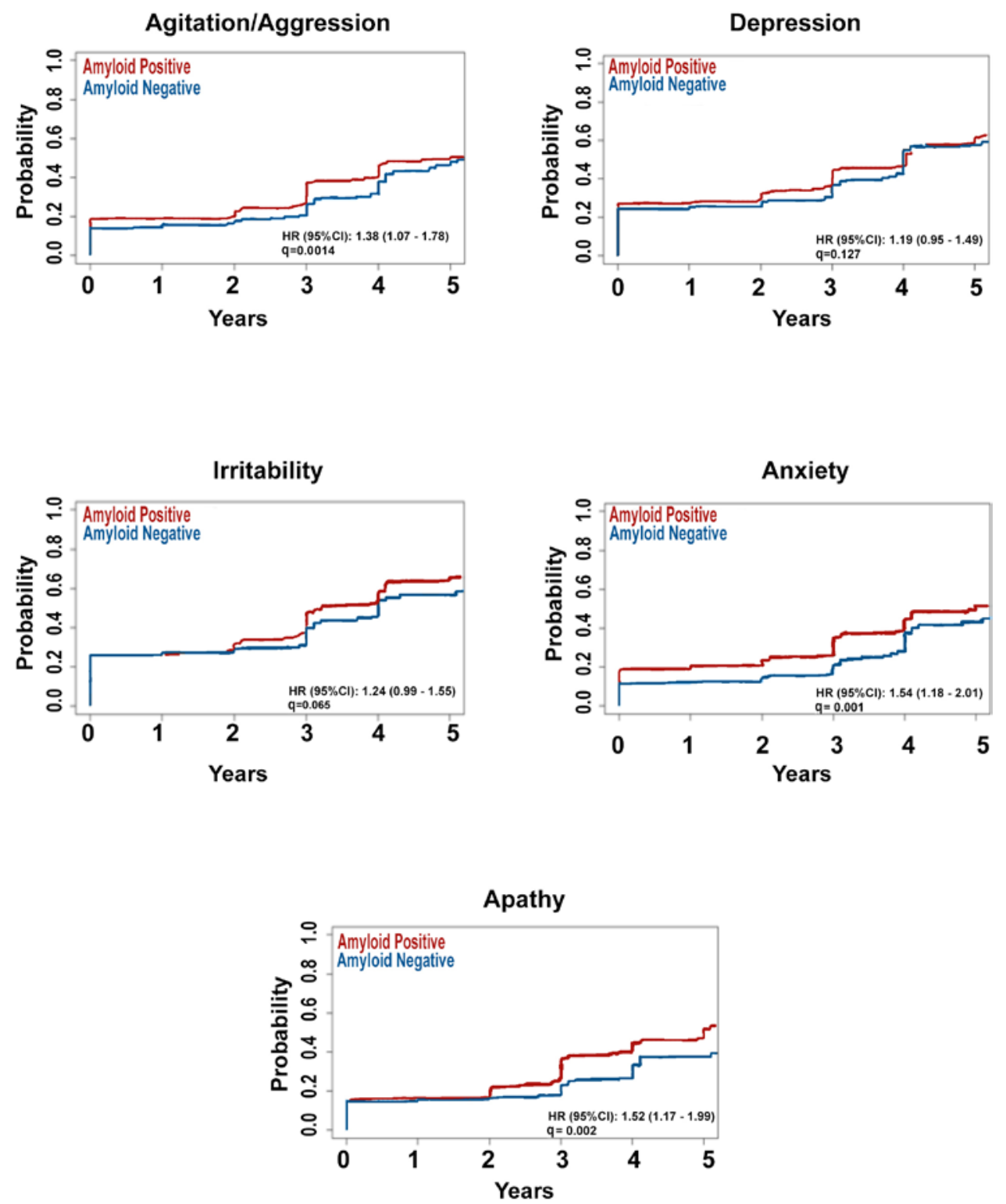

Figure 2 Survival curves showing time to emergence of the five most frequent early neuropsychiatricsymptoms in mild cognitive impairment by amyloid status.

- delusions, hallucinations, agitation, anxiety, apathy, irritability, depression, euphoria, disinhibition, aberrant motor behaviour, sleep and appetite. Interviewers ask structured questions about the presence and severity (as well as frequency in the full version of the NPI, but not in NPI-Q) of the symptoms in the past month to the study partner. Those enrolled in ADNI-1 only had the NPI-Q available, while those enrolled in ADNI-2 and ADNI-GO were administered the full version.

NPI or NPI-Q data from the baseline and all annual visits were obtained from the Laboratory of Neuroimaging Image Data Archive (LONI IDA) on 3 November, 2015. Each patient had either NPI or NPI-Q for each visit that was analysed. NPS at baseline were coded as 'absent' if not endorsed and as 'present' if endorsed by the study partner. In our longitudinal analyses, we coded symptoms in follow-up as 'absent' if never endorsed by the study partner (including the baseline visit) and as 'emerging de novo' if absent at baseline but reported at one or more follow-up visit. Frequency x severity (FxS) scores were obtained for subjects that received the full NPI questionnaire at baseline.

\section{Imaging data and analysis}

A detailed description of AV-45 PET acquisition may be found at http://www.adni-info.org/Scientists/ADNIStudyProcedures.html. Briefly, $370 \mathrm{MBq}(10 \mathrm{mCi}+/-10 \%)$ bolus injection of AV-45 was administered intravenously. Approximately $50 \mathrm{~min}$ after injection, a $20 \mathrm{~min}$ continuous brain PET imaging session collected a dynamic amyloid PET data consisting of four $5 \mathrm{~min}$ frames. In our main analyses, we used the mean whole brain standard uptake volume ratios (SUVR) from University of California, Berkeley (UCB), downloaded from ADNI's database on 3 November, 2015. The mean whole brain SUVR was obtained by averaging the SUVR values across 

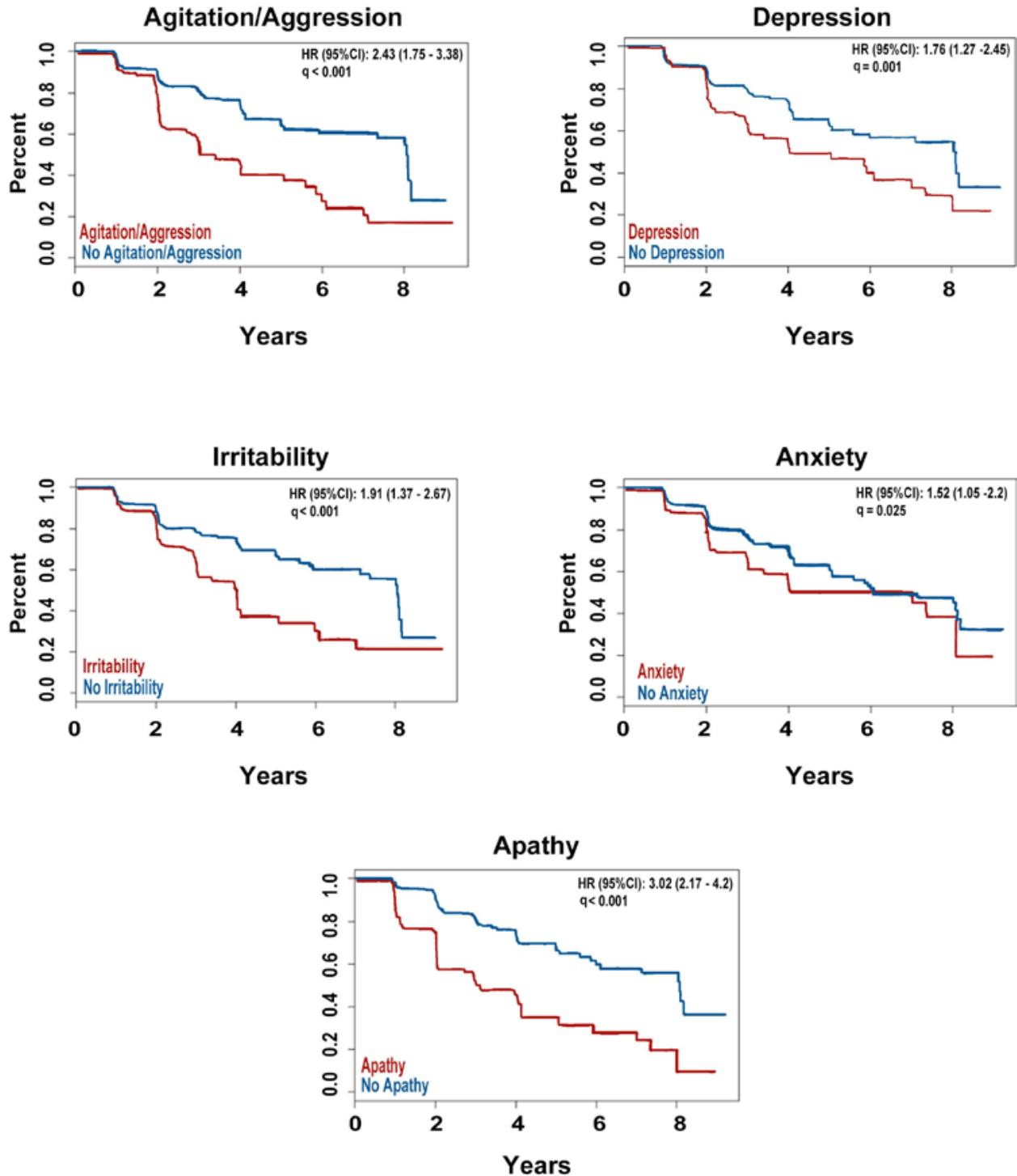

Figure 3 Survival curves showing the effect of the presence of the five most frequent early neuropsychiatricsymptoms on time to conversion from mild cognitive impairment to dementia.

the frontal, anterior/posterior cingulate, lateral parietal and lateral temporal grey matter regions. ${ }^{40}$ The UCB protocols for $\left({ }^{18} \mathrm{~F}\right)$-florbetapir preprocessing, co-registration and normalisation have been previously described. ${ }^{40}$ We defined a positive amyloid PET scan as mean SUVR $\geq 1.17 .^{41}$

\section{Statistical analyses}

Demographic comparisons between amyloid positive and amyloid negative groups within diagnostic categories were done using Fisher's exact for categorical variables and Wilcoxon rank-sum test for continuous variables. Comparisons of the frequency of symptoms at baseline, as well as the emergence of new NPS in follow-up conditional on amyloid status were done using Fisher's exact test. Using stepwise backwards logistical regression we also studied the predictive value of the presence/absence of NPI behaviours as well as their FxS scores on amyloid status while adjusting for age, sex, education and ApolipoproteinE $\varepsilon 4$ (APOE4).
Survival analyses using Cox proportional hazard regression models were used to determine (1) the hazard ratios for developing the five most common early NPI symptoms - apathy, anxiety, agitation, irritability and depression - in amyloid positive versus amyloid negative participants in the NC, SMC and MCI groups; (2) the hazard for conversion from MCI to dementia in all MCI subjects based on the presence of the top five most common early NPI symptoms at baseline and (3) the hazard for conversion from MCI to dementia in MCI subjects who were free of the five most common NPI symptoms at baseline but developed them in follow-up. For analyses (2) and (3), subsequent visits were excluded once a subject was diagnosed with dementia. Subjects who reverted from MCI to NC $(n=34)$ in follow-up were excluded from all analyses. We censored the data ignoring visits after dementia was diagnosed in our time to conversion Cox proportional hazard regression models. All Cox proportional hazard regression models were adjusted for age, education and 

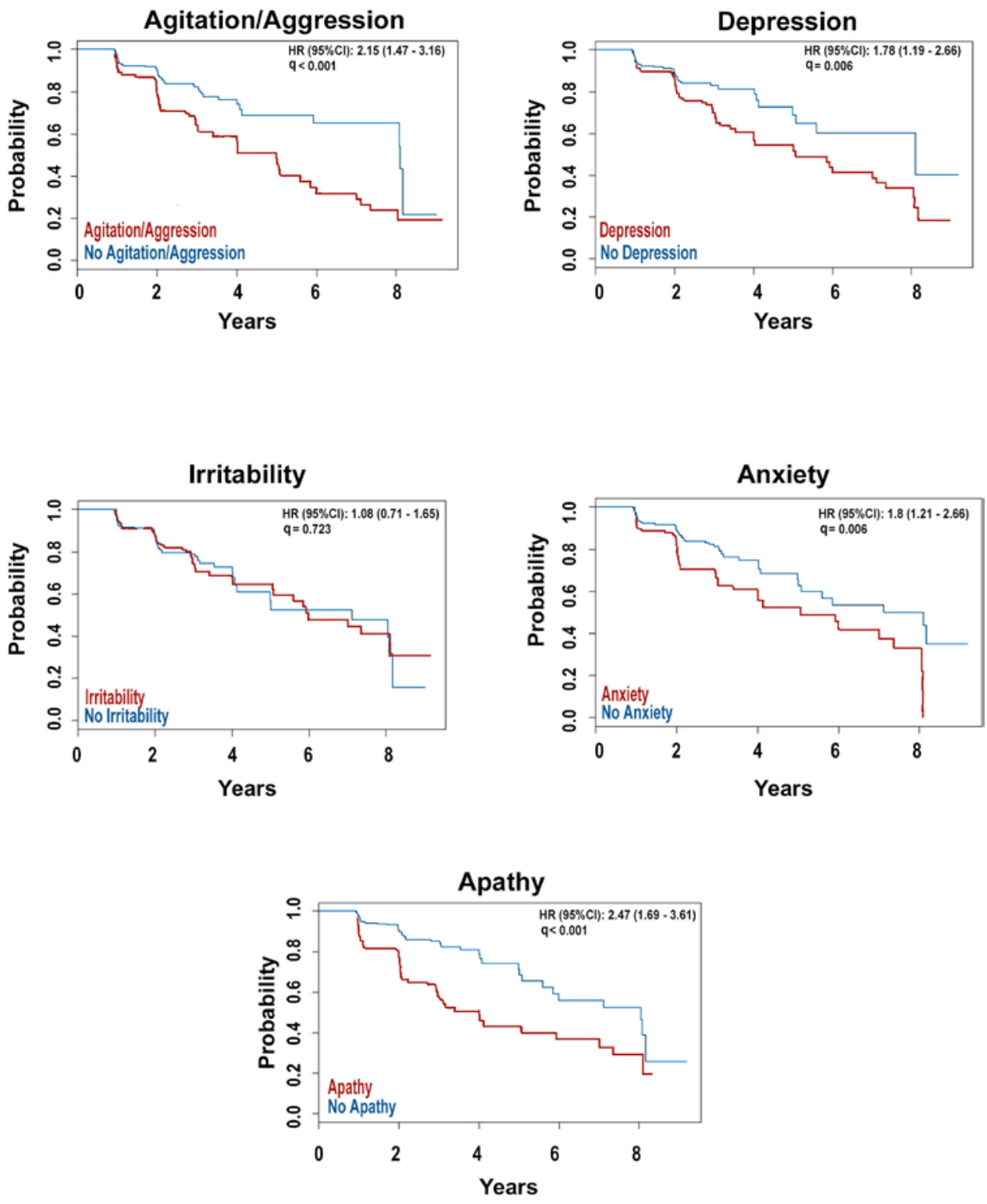

Figure 4 Survival curves showing the effect of de novo development of the five most frequent early neuropsychiatric symptoms on time to conversion from mild cognitive impairment to dementia.

APOE4 status. The Cox regression models were repeated while additionally adjusting for amyloid status. P values were adjusted for multiple comparisons using false discovery rate (FDR). All Cox regression models were evaluated for proportional hazard assumption and there was no evidence that the models did not meet required assumptions ( $q$-value $>0.081$ ). All $p$ values were adjusted for multiple comparison correction using Benjamini \& Hochberg ${ }^{42}$ FDR correction. All statistical tests were twosided and a q-value less than 0.05 was considered statistically significant. All statistical analyses were done in the R Statistical Computing Environment (R Core Team, Vienna, Austria).

\section{Patient and public involvement}

Our study represents secondary analyses of preexisting data. Patients or the public were not engaged in defining our research questions and outcome measures and have not provided input into study design and conduct of this study. A yearly manuscript summarising all findings from the ADNI study is published by the ADNI leadership team. ${ }^{43-45}$ As our analyses use deidentified data only, study participants will not be contacted with these findings.

\section{RESULTS}

Our sample consisted of a total of 1077 ADNI subjects including $275 \mathrm{NC}$ (26.5\% amyloid positive), $100 \mathrm{SMC}$ (28.0\% amyloid positive), 559 MCI (52.0\% amyloid positive) and $143 \mathrm{AD}$ (82.5\% amyloid positive) subjects with average follow-up time of 3.2 \pm 2.4 years (table 1 ). Amyloid positive $\mathrm{NC}$ and SMC were significantly older and more likely to be APOE4 positive relative to their amyloid negative counterparts. Amyloid positive NC were also less educated and performed worse on the Alzheimer's 
Disease Assessment Scale - Cognition Subscale 11 (ADAScog-11) compared with amyloid negative NC. Amyloid positive MCI were significantly older, more likely to be APOE4 positive, and showed greater impairment on MMSE, Clinical Dementia Rating - Sum of Boxes, ADAScog-11 and Functional Assessment Questionnaire scales compared with amyloid negative MCI. Amyloid positive dementia subjects were more likely to be APOE4 positive, and showed greater impairment on the ADAScog-11 compared with the amyloid negative dementia subgroup. No significant difference in years of follow-up was seen between the amyloid positive versus negative subjects in each of the diagnostic groups (table 1).

\section{Neuropsychiatric symptoms at baseline}

No differences in NPS frequency at baseline were seen between amyloid positive and amyloid negative NC, SMC, MCI or dementia groups (online supplementary table 1). Comparing NPI FxS means between the amyloid positive and negative subgroups within each diagnostic group revealed significantly greater aberrant motor behaviours FxS in amyloid positive versus amyloid negative $\mathrm{NC}(0.083$ vs $0.005, \mathrm{p}=0.04$ ). Compared with amyloid negative MCI, amyloid positive MCI manifested significantly greater anxiety FxS (0.569 vs 0.314, p=0.023), delusions FxS (0.138 vs $0.004, \mathrm{p}=0.001)$ and aberrant motor behaviours FxS (0.286 vs $0.088, p=0.025)$. Compared with amyloid negative dementia subjects, amyloid positive dementia cases showed significantly lower apathy $\mathrm{FxS}$ (1.271 vs 2.583, $\mathrm{p}=0.011)$, agitation FxS (0.619 vs $1.708, \mathrm{p}=0.003)$ and appetite changes $(0.864$ vs $2.417, \mathrm{p}=0.006)$, however these findings should be cautiously interpreted due to the very small sample size of the amyloid negative dementia group ( $\mathrm{n}=25 \mathrm{vs} \mathrm{n}=118$ amyloid positive). No significant differences were seen in SMC. The full statistical models can be seen in online supplementary table 2 .

\section{Cumulative incidence of NPS in follow-up}

No differences in the rates of de novo development of NPS were seen between amyloid positive and amyloid negative subjects in the NC, SMC or dementia groups. New onset delusions $(13.4 \%$ vs $2.2 \%, \mathrm{p}<0.001)$, hallucinations $(8.0 \%$ vs $2.2 \%, \mathrm{p}=0.007)$, anxiety $(35.9 \%$ vs $24.8 \%$, $\mathrm{p}=0.014)$, apathy $(38.4 \%$ vs $21.8 \%, \mathrm{p}<0.001)$, disinhibition $(24.2 \%$ vs $14.7 \%, \mathrm{p}=0.014)$, irritability $(45.5 \%$ vs $32.7 \%, \mathrm{p}=0.014)$, aberrant motor disturbances $(18.1 \%$ vs $9.2 \%, \mathrm{p}=0.008)$ and appetite disturbances $(33.5 \%$ vs $20.9 \%, \mathrm{p}=0.007$ ) were significantly more common in amyloid positive versus amyloid negative MCI (figure 1). The full statistical models can be seen in online supplementary table 3 .

\section{Predictors of amyloid status at baseline}

In our pooled stepwise backwards logistic regression model, anxiety ( $\mathrm{OR}=1.87,95 \% \mathrm{CI} 1.17$ to $3.02, \mathrm{p}=0.018)$ and delusions $(\mathrm{OR}=7.01,95 \%$ CI 1.25 to $132.05, \mathrm{p}=0.04)$ were predictive of amyloid positivity after adjusting for age, sex, education and APOE4 in the pooled sample.
When we analysed the same relationship using the continuous $\mathrm{FxS}$ measure, delusions were a significant predictor $(\mathrm{OR}=2.22,95 \%$ CI 1.18 to $6.23, \mathrm{p}=0.012)$.

Among MCI participants the presence of anxiety $(\mathrm{OR}=1.77,95 \% \mathrm{CI} 1.03$ to $3.09, \mathrm{p}=0.04)$ at baseline was predictive of $\mathrm{AD}$ pathology. Using the continuous $\mathrm{FxS}$ measure revealed significant association between delusions and amyloid pathology (OR=5.8, 95\% CI 1.69 to 98.36, $\mathrm{p}=0.001$ ).

No significant differences were seen among the individual NC, SMC or dementia groups.

\section{Time to development of the five most frequent early NPS by amyloid status}

Here we focused on the five most common early NPS apathy, anxiety, agitation, irritability and depression. Time to de novo development of these NPS in NC and SMC did not differ by amyloid status. After adjusting for age, education and APOE4 status, amyloid pathology in MCI was associated with faster emergence of agitation $(\mathrm{HR}=1.47$, $95 \%$ CI 1.09 to $1.99, \mathrm{p}=0.012)$, anxiety $(\mathrm{HR}=1.45,95 \% \mathrm{CI}$ 1.07 to $1.98, \mathrm{p}=0.017)$ and apathy $(\mathrm{HR}=1.34,95 \%$ CI 0.98 to $1.83, \mathrm{p}<0.001$ ) (figure 2).

\section{Effect of baseline NPS on time to conversion in MCI}

Individually, adjusting for age, education and APOE4 status, four of the five early NPS at baseline were significantly associated with time to conversion from MCI to dementia (agitation $\mathrm{HR}=2.28$, 95\% CI 1.64 to 3.17, $\mathrm{p}<0.001$; depression $\mathrm{HR}=1.51,95 \%$ CI 1.08 to 2.11 , $\mathrm{p}=0.021$; irritability $\mathrm{HR}=1.88$, 95\% CI 1.35 to 2.63, $\mathrm{p}<0.001$; anxiety $\mathrm{HR}=1.39,95 \%$ CI 0.96 to $2.02, \mathrm{p}=0.083$; apathy $\mathrm{HR}=2.69,95 \% \mathrm{CI} 1.92$ to $3.78, \mathrm{p}<0.001)$. Additionally, adjusting for amyloid status did not change the results (agitation $\mathrm{HR}=1.78,95 \%$ CI 1.27 to 2.49 , $\mathrm{p}=0.002$; depression HR=1.44, 95\% CI 1.03 to 2.02, $\mathrm{p}=0.04$; irritability $\mathrm{HR}=1.61,95 \%$ CI 1.15 to $2.25, \mathrm{p}=0.009$; anxiety $\mathrm{HR}=1.2,95 \%$ CI 0.82 to $1.75, \mathrm{p}=0.341$; apathy $\mathrm{HR}=2.57$, $95 \%$ CI 1.83 to $3.61, \mathrm{p}<0.001$ ).

When all five symptoms were entered simultaneously into a multivariable Cox regression model adjusting for age, education and APOE4 status agitation and apathy remained significant (agitation $\mathrm{HR}=1.76$, 95\% CI 1.19 to 2.6, $\mathrm{p}=0.005$; apathy $\mathrm{HR}=2.59,95 \%$ CI 1.81 to 3.71 , $\mathrm{p}<0.001)$. After additionally adjusting for baseline amyloid status apathy remained significant (agitation $\mathrm{HR}=1.46,95 \%$ CI 0.99 to 2.15 , $\mathrm{p}=0.057$; apathy $\mathrm{HR}=2.48$, $95 \%$ CI 1.73 to $3.56, \mathrm{p}<0.001$ ) (figure 3 ).

\section{Effect of de novo NPS on time to conversion in MCI}

Individually, adjusting for age, education and APOE4 status, the emergence of agitation, depression, anxiety and apathy were associated with greater risk for conversion from MCI to dementia (agitation $\mathrm{HR}=2.17,95 \%$ CI 1.47 to $3.2, \mathrm{p}<0.001$; depression $\mathrm{HR}=1.54,95 \%$ CI 1.02 to $2.33, \mathrm{p}=0.049$; anxiety $\mathrm{HR}=2.17,95 \%$ CI 1.47 to 3.2 , $\mathrm{p}<0.001$; apathy $\mathrm{HR}=2.33,95 \%$ CI 1.57 to $3.45, \mathrm{p}<0.001$ ). Agitation, anxiety and apathy remained significant when 
we also adjusted for amyloid status (agitation $\mathrm{HR}=2.03$, $95 \% \mathrm{CI} 1.37$ to $3, \mathrm{p}=0.001$; anxiety $\mathrm{HR}=2.03,95 \% \mathrm{CI}$ 1.37 to $3, \mathrm{p}=0.001$; apathy $\mathrm{HR}=2.42,95 \%$ CI 1.65 to 3.57 , $\mathrm{p}<0.001)$.

When all five symptoms were entered simultaneously into a multivariable Cox regression model adjusting for age, education and $A P O E 4$ status, de novo agitation, irritability and apathy were significant predictors of conversion from MCI to dementia (agitation $\mathrm{HR}=2.0,95 \%$ CI 1.17 to 3.43, $\mathrm{p}=0.012$; irritability HR $=0.45,95 \%$ CI 0.24 to 0.82 , $\mathrm{p}=0.009$; apathy $\mathrm{HR}=2.09,95 \%$ CI 1.17 to $3.73, \mathrm{p}=0.012$ ). Additionally, adjusting for baseline amyloid status, did not change the results (agitation HR=1.91, 95\% CI 1.12 to 3.28 , $\mathrm{p}=0.018$; irritability $\mathrm{HR}=0.47,95 \%$ CI 0.26 to 0.86 , $\mathrm{p}=0.014$; apathy $\mathrm{HR}=2.42$, $95 \%$ CI 1.39 to 4.23 , $\mathrm{p}=0.002$ ) (figure 4).

\section{DISCUSSION}

Here we investigated the relationship between amyloid burden and incidence and frequency of NPS across the spectrum from normal cognition to dementia. We hypothesised that amyloid pathology will associate with higher frequency of NPS across all disease stages, but we found that while the frequency of NPS is not significantly different between amyloid negative and positive diagnostic groups, some NPS might be more severe in those who are amyloid positive (online supplementary table 2). This held true for aberrant motor behaviours among amyloid positive NC and MCI, as well as anxiety and delusions in amyloid positive MCI. Our findings in the MCI group is in line with those by Krell-Roesch et al, 2019, who found that compared with MCI and NC without amyloid burden, MCI with amyloid burden but not NC with amyloid burden had an increased risk of having NPS. ${ }^{26}$ Looking into whether neuropsychiatric symptoms carry prognostic value of Alzheimer's pathology we found anxiety to be predictive of brain amyloidosis in the pooled and MCI samples. Delusions carried additional predictive value in MCI.

In line with our hypothesis, a greater proportion of the amyloid positive MCI had emergence of delusions, hallucinations, anxiety, apathy, disinhibition, irritability, aberrant motor behaviours and appetite changes compared with amyloid negative MCI over the course of the study (figure 1). These findings are as expected considering the prominence and progressive development of NPS over time during the course of the disease. ${ }^{67}$ Our Cox proportional hazard regression model further demonstrated that participants with MCI due to AD develop apathy, anxiety and agitation - three of the earliest and most pervasive NPS in the MCI stage, earlier compered to amyloid negative MCI (figure 2). ${ }^{6}$

Contrary to our expectations we found that amyloid negative dementia subjects have higher frequency of apathy, agitation and appetite changes compared with the amyloid positive dementia group. One possible explanation is that previous studies on the prevalence of NPS in $\mathrm{AD}$ dementia have not included biomarker validation. This means that $\mathrm{AD}$ phenocopies with amnestic presentation were included as $\mathrm{AD}$ cases. ADNI is the first large scale observational study that included amyloid PET as a biomarker. Thus, for the first time we have the opportunity to investigate the frequency of NPS in biomarker validated $\mathrm{AD}$ dementia and compare that to $\mathrm{AD}$ phenocopies. What we find here suggests that these previous reports might have overestimated the true prevalence of some NPS in AD dementia due to including amyloid negative $\mathrm{AD}$ phenocopies in the $\mathrm{AD}$ dementia group. An alternative explanation is that ADNI subjects who are amyloid negative have other neurodegenerative disorders such as frontotemporal dementia, dementia with Lewy bodies, argyrophilic grain disease, hippocampal sclerosis, etc. These other pathological entities could also explain the increased frequencies of NPS.

The survival analyses looking into the effects of the five most frequent early NPS on time to conversion from MCI to dementia showed that four of the five early NPS symptoms - agitation, apathy, depression and irritability, were individually predictive of conversion from MCI to dementia as previously reported. ${ }^{10}{ }^{13-19}$ Of these agitation and apathy remained significant when adjusting for the presence of all five behaviours as previously reported. ${ }^{131417}$

When we focused on the predictive effects of newly emerging symptoms on future conversion from MCI to dementia, four of the five symptoms - agitation, depression, anxiety and apathy, were individually predictive. Agitation, irritability and apathy proved to be the main drivers of this relationship when adjusting for the presence of all five behaviours.

Taken together our data seem to indicate that both prevalent agitation, and apathy, and incident agitation, irritability and apathy are predictive of faster functional decline and loss of independence among amyloid positive and negative MCI. Our findings regarding agitation seem to agree with those reported by Brodaty $e t \mathrm{al}^{13}$ who also found that agitation was significantly associated with cognitive decline. Several studies have also concluded that apathy is a useful NPS for identifying cognitive decline to $\mathrm{AD}{ }^{1846}$ Affective symptoms, including irritability, have also been previously reported in association with later cognitive decline to dementia. ${ }^{47}$

Contrary to our expectations and the observations of others ${ }^{1130}$ NPS were not associated with faster cognitive decline among ADNI's amyloid positive NC. We could hypothesise that both the sample size differences $(n=275$ in our study vs $n=1587$ in the large population-based study by Geda $e t a l^{11}$ ) as well as the different inclusion criteria used by these two studies (ie, the mandatory lack of even perceived age-associated decline and the exclusion of individuals with baseline depression defined as Getriatric Depression Scale (GDS) $>5$ in ADNI NC) and the fact that Geda $e t$ al did not have information regarding brain amyloidosis, as potential drivers for these differences.

Several strengths and limitations of the ADNI study should be acknowledged. One of the strengths of this 
study is our cohort. ADNI is the premier longitudinal biomarker study in AD. ADNI employs unified subject assessment and PET scan collection protocols and meticulous data quality control across all study sites. Another strength of our study is the relatively large sample size and the inclusion of all diagnostic groups across the $\mathrm{AD}$ spectrum instead of just the symptomatic ones as it has been done in the past. Another strength of our paper is the use of neuropsychiatric data collection tools that are administered to the caregivers and not the subject themselves. One of the limitations of this study is that the NPI and NPI-Q use structured questions focused on the presence and severity of symptoms from the preceding month only, and therefore, intermittent NPS that were not manifested by the subjects in the prespecified timeframe were not captured. Another limitation to our study is that ADNI employs rigorous exclusion criteria typical of clinical trials and the study population and hence our study cohort might not be representative of the general population. Moreover, ADNI criteria excludes subjects with GDS greater than or equal to five and any primary psychiatric condition. These selection criteria undoubtedly have further influenced on our study and might limit the overall generalisability of our findings to the elderly population as a whole. Lastly, since the model selection and model building were done in the same data set, our results are considered explanatory. Further studies are warranted to confirm our results.

In summary, we investigated the relationship between amyloid burden and occurrence of NPS in elderly with and without cognitive decline at baseline and over time. We found that amyloid pathology is a significant risk factor for future development of NPS in MCI, but not in the presymptomatic or at-risk stages of the disease. We also found that the presence of apathy, agitation, depression and irritability in MCI patients predict a more aggressive disease course regardless of the presence or absence of amyloid pathology.

\section{Author affiliations \\ ${ }^{1}$ Robert Larner College of Medicine, Burlington, Vermont, USA \\ ${ }^{2}$ Neurology, Emory University School of Medicine, Atlanta, Georgia, USA \\ ${ }^{3}$ Medicine Statistics Core, University of California Los Angeles, Los Angeles, \\ California, USA \\ ${ }^{4}$ Tulane University School of Medicine, New Orleans, Louisiana, USA \\ ${ }^{5}$ Neurology, Indiana University School of Medicine, Indianapolis, Indiana, USA \\ ${ }^{6}$ Psychiatry, Indiana University School of Medicine, Indianapolis, Indiana, USA}

Acknowledgements We would like to acknowledge and thank Dr David Elashoff from the Department of Medicine Statistics Core at the University of California, Los Angeles, for his assistance with the statistical design of this manuscript.

Contributors NG assisted with data processing and analyses, and the completion of integral statistical analyses. NG was the primary author responsible for drafting the manuscript. KSH assisted with data processing and analyses, provided critical insights for interpretation of the results and participated in revising of the manuscript. TR completed integral statistical analyses, provided critical insights for interpretation of our results and participated in revising of the manuscript. JG completed integral statistical analyses, provided critical insights for interpretation of our results and participated in revising of the manuscript. TMD completed some of the analyses and took part in revising of the manuscript. JG assisted in editing of figures and participated in revising of the manuscript. DRB provided critical insights for interpretation of our results and participated in revising of the manuscript. LGA was responsible for the study concept and design. She provided significant oversight over all analyses, interpretation of results and participated in all stages of manuscript preparation.

Funding This work was generously supported by NIA R01 AG040770, NIA K02 AG048240, NIA P50 AG16570, NIA P30 AG010133, NIA U01 AG024904 and the Easton Consortium for Alzheimer's Drug Discovery and Biomarker Development. Data collection and sharing for this project was funded by the Alzheimer's Disease Neuroimaging Initiative (ADNI) (National Institutes of Health Grant U01 AG024904) and DOD ADNI (Department of Defense award number W81XWH-12- 2-0012). ADNI is funded by the National Institute on Aging, the National Institute of Biomedical Imaging and Bioengineering and through generous contributions from the following: AbbVie, Alzheimer's Association; Alzheimer's Drug Discovery Foundation; Araclon Biotech; BioClinica, Inc; Biogen; Bristol-Myers Squibb Company; CereSpir, Inc; Cogstate; Eisai Inc; Elan Pharmaceuticals, Inc; Eli Lilly and Company; Eurolmmun; F. Hoffmann-La Roche Ltd and its affiliated company Genentech, Inc; Fujirebio; GE Healthcare; IXICO Ltd; Janssen Alzheimer Immunotherapy Research \& Development, LLC; Johnson \& Johnson Pharmaceutical Research \& Development LLC; Lumosity; Lundbeck; Merck \& Co, Inc; Meso Scale Diagnostics, LLC; NeuroRx Research; Neurotrack Technologies; Novartis Pharmaceuticals Corporation; Pfizer Inc; Piramal Imaging; Servier; Takeda Pharmaceutical Company and Transition Therapeutics. The Canadian Institutes of Health Research is providing funds to support ADNI clinical sites in Canada. Private sector contributions are facilitated by the Foundation for the National Institutes of Health (https://fnih.org/). The grantee organisation is the Northern California Institute for Research and Education, and the study is coordinated by the Alzheimer's Therapeutic Research Institute at the University of Southern California. ADNI data are disseminated by the Laboratory for Neuro Imaging at the University of Southern California.

Competing interests Liana G Apostolova received research support from General Electric Healthcare, Piramal and Eli Lilly, served on the speaker's bureau for Eli Lilly \& Company and Piramal Enterprises and on an advisory board for Eli Lilly \& Company.

Patient consent for publication Not required.

Provenance and peer review Not commissioned; externally peer reviewed.

Open access This is an open access article distributed in accordance with the Creative Commons Attribution Non Commercial (CC BY-NC 4.0) license, which permits others to distribute, remix, adapt, build upon this work non-commercially, and license their derivative works on different terms, provided the original work is properly cited, appropriate credit is given, any changes made indicated, and the use is non-commercial. See: http://creativecommons.org/licenses/by-nc/4.0/.

\section{ORCID iD}

Naira Goukasian http://orcid.org/0000-0002-6765-2201

\section{REFERENCES}

1 Barthel H, Gertz H-J, Dresel S, et al. Cerebral amyloid- $\beta$ PET with florbetaben (18F) in patients with Alzheimer's disease and healthy controls: a multicentre phase 2 diagnostic study. The Lancet Neurology 2011;10:424-35.

2 Clark CMet al. Use of Florbetapir-PET for imaging $\beta$-amyloid pathology. JAMA 2011;305:275-83.10.1001/jama.2010.2008

3 Vandenberghe R, Van Laere K, Ivanoiu A, et al. ${ }^{18}$ F-flutemetamol amyloid imaging in Alzheimer disease and mild cognitive impairment: A phase 2 trial. Ann Neurol 2010;68:319-29.

4 Dubois B, Hampel H, Feldman HH, et al. Preclinical Alzheimer's disease: definition, natural history, and diagnostic criteria. Alzheimers Dement 2016;12:292-323.

5 Sperling R, Mormino E, Johnson K. The Evolution of Preclinical Alzheimer's Disease: Implications for Prevention Trials. Neuron 2014;84:608-22.

6 Apostolova LG, Cummings JL. Neuropsychiatric manifestations in mild cognitive impairment: a systematic review of the literature. Dement Geriatr Cogn Disord 2008;25:115-26.

7 Mega MS, Cummings JL, Fiorello T, et al. The spectrum of behavioral changes in Alzheimer's disease. Neurology 1996;46:130-5.

8 Steinberg M, Shao H, Zandi P, et al. Point and 5-year period prevalence of neuropsychiatric symptoms in dementia: the Cache County study. Int J Geriatr Psychiatry 2008;23:170-7.

9 Lyketsos CGet al. Mental and behavioral disturbances in dementia: findings from the Cache County study on memory in aging. $\mathrm{Am} \mathrm{J}$ Psychiatry 2000;157:708-14. 
10 Donovan NJ, Amariglio RE, Zoller AS, et al. Subjective cognitive concerns and neuropsychiatric predictors of progression to the early clinical stages of Alzheimer disease. Am J Geriatr Psychiatry 2014:22:1642-51.

11 Geda YE, Roberts RO, Mielke MM, et al. Baseline neuropsychiatric symptoms and the risk of incident mild cognitive impairment: a population-based study. AJP 2014:171:572-81.

12 Zufferey V, Donati A, Popp J, et al. Neuroticism, depression, and anxiety traits exacerbate the state of cognitive impairment and hippocampal vulnerability to Alzheimer's disease. Alzheimers Dement 2017;7:107-14

13 Brodaty $\mathrm{H}$, Heffernan M, Draper B, et al. Neuropsychiatric symptoms in older people with and without cognitive impairment. $J A D$ 2012;31:411-20.

14 Palmer K, Di lulio F, Varsi AE, et al. Neuropsychiatric predictors of progression from amnestic-mild cognitive impairment to Alzheimer's disease: the role of depression and apathy. JAD 2010;20:175-83.

15 Pink A, Stokin GB, Bartley MM, et al. Neuropsychiatric symptoms, APOE 4, and the risk of incident dementia: A population-based study. Neurology 2015;84:935-43.

16 Rosenberg PB, Mielke MM, Appleby BS, et al. The association of neuropsychiatric symptoms in $\mathrm{MCl}$ with incident dementia and Alzheimer disease. Am J Geriatr Psychiatry 2013;21:685-95.

17 Somme J, Fernandez-Martinez M, Molano A, et al. Neuropsychiatric symptoms in amnestic mild cognitive impairment: increased risk and faster progression to dementia. Curr Alzheimer Res 2013;10:86-94.

18 Teng E, Lu PH, Cummings JL. Neuropsychiatric Symptoms Are Associated with Progression from Mild Cognitive Impairment to Alzheimer's Disease. Dement Geriatr Cogn Disord 2007;24:253-9.

19 Wadsworth LP, Lorius N, Donovan NJ, et al. Neuropsychiatric symptoms and global functional impairment along the Alzheimer's continuum. Dement Geriatr Cogn Disord 2012;34:96-111.

20 Peters ME, Schwartz S, Han D, et al. Neuropsychiatric Symptoms as Predictors of Progression to Severe Alzheimer's Dementia and Death: The Cache County Dementia Progression Study. AJP 2015;172:460-5.

21 Rabins PV, Schwartz S, Black BS, et al. Predictors of progression to severe Alzheimer's disease in an incidence sample. Alzheimers Dement 2013:9:204-7.

22 Fischer CE, Ismail Z, Schweizer TA. Impact of neuropsychiatric symptoms on caregiver burden in patients with Alzheimer's disease. Neurodegener Dis Manag 2012;2:269-77.

23 Cepoiu-Martin M, Tam-Tham H, Patten S, et al. Predictors of longterm care placement in persons with dementia: a systematic review and meta-analysis. Int J Geriatr Psychiatry 2016;31:1151-71.

24 Gaugler JE, Yu F, Krichbaum K, et al. Predictors of nursing home admission for persons with dementia. Med Care 2009;47:191-8.

25 Geda YE, Schneider LS, Gitlin LN, et al. Neuropsychiatric symptoms in Alzheimer's disease: past progress and anticipation of the future. Alzheimer's \& Dementia 2013;9:602-8.

26 Krell-Roesch J, Vassilaki M, Mielke MM, et al. Cortical $\beta$-amyloid burden, neuropsychiatric symptoms, and cognitive status: the Mayo clinic study of aging. Trans/ Psychiatry 2019;9:123.

27 Marshall GA, Donovan NJ, Lorius N, et al. Apathy is associated with increased amyloid burden in mild cognitive impairment. $J$ Neuropsychiatry Clin Neurosci 2013;25:302-7.

28 Ramakers IHGB, Verhey FRJ, Scheltens P, et al. Anxiety is related to Alzheimer cerebrospinal fluid markers in subjects with mild cognitive impairment. Psychol Med 2013;43:911-20.
29 Bensamoun D, Guignard R, Furst AJ, et al. Associations between neuropsychiatric symptoms and cerebral amyloid deposition in cognitively impaired elderly people. JAD 2016;49:387-98.

30 Pietrzak RH, Lim YY, Neumeister A, et al. Amyloid-Beta, anxiety, and cognitive decline in preclinical Alzheimer disease: a multicenter, prospective cohort study. JAMA Psychiatry 2015;72:284-91.

31 Babulal GM, Ghoshal N, Head D, et al. Mood changes in cognitively normal older adults are linked to Alzheimer disease biomarker levels. Am J Geriatr Psychiatry 2016;24:1095-104.

32 Petersen RC, Aisen PS, Beckett LA, et al. Alzheimer's disease neuroimaging Initiative (ADNI): clinical characterization. Neurology 2010;74:201-9.

33 McKhann G, Drachman D, Folstein M, et al. Clinical diagnosis of Alzheimer's disease: report of the NINCDS-ADRDA work Group* under the auspices of department of health and human services Task force on Alzheimer's disease. Neurology 1984;34:939-44.

34 Folstein MF, Folstein SE, McHugh PR, et al. A practical method for grading the cognitive state of patients for the clinician. J Psychiatr Res 1975;12:189-98.

35 Morris JC. The clinical dementia rating (CDR): current version and scoring rules. Neurology 1993;43:2412-4.

36 Wechsler D. WMS-R: Wechsler memory Scale-Revised manual. San Antonio, tx: psychological Corp. Harcourt Brace Jovanovich 1987.

37 Saykin AJ, Wishart HA, Rabin LA, et al. Older adults with cognitive complaints show brain atrophy similar to that of amnestic $\mathrm{MCl}$. Neurology 2006;67:834-42.

38 Cummings JL. The neuropsychiatric inventory: assessing psychopathology in dementia patients. Neurology 1997;48:10S-16.

39 Kaufer DI, Cummings JL, Ketchel P, et al. Validation of the NPI-Q, a brief clinical form of the neuropsychiatric inventory. $J$ Neuropsychiatry Clin Neurosci 2000;12:233-9.

40 Jagust WJ, Bandy D, Chen K, et al. The Alzheimer's disease neuroimaging initiative positron emission tomography core. Alzheimers Dement 2010;6:221-9.

41 Fleisher ASet al. Using positron emission tomography and florbetapir $\mathrm{F} 18$ to image cortical amyloid in patients with mild cognitive impairment or dementia due to Alzheimer disease. Arch Neurol 2011;68:1404-11.

42 Benjamini Y, Hochberg Y. Controlling the false discovery rate: a practical and powerful approach to multiple testing. J Royal Statist Soc 1995;57:289-300.

43 Weiner MW, Veitch DP, Aisen PS, et al. Impact of the Alzheimer's disease neuroimaging initiative, 2004 to 2014. Alzheimers Dement 2015;11:865-84.

44 Weiner MW, Veitch DP, Aisen PS, et al. 2014 update of the Alzheimer's disease neuroimaging Initiative: a review of papers published since its inception. Alzheimers Dement 2015;11:e1-120.

45 Weiner MW, Veitch DP, Aisen PS, et al. Recent publications from the Alzheimer's disease neuroimaging Initiative: reviewing progress toward improved AD clinical trials. Alzheimers Dement 2017;13:e1-85.

46 Starkstein SEet al. A prospective longitudinal study of apathy in Alzheimer's disease. J Neurol Neurosurg Psychiat 2006;77:8-11.

47 Ismail Z, Gatchel J, Bateman DR, et al. Affective and emotional dysregulation as pre-dementia risk markers: exploring the mild behavioral impairment symptoms of depression, anxiety, irritability, and euphoria - CORRIGENDUM. Int Psychogeriatr 2019;31. 